\title{
Exploitation of rumen microbial enzymes to benefit ruminant production
}

\author{
KJ Cheng 1, TA McAllister 1, LB Selinger 1, LJ Yanke 1, \\ HD Bea 1, CW Forsberg 2, JA Shelford ${ }^{3}$ \\ ' Research Centre, Agriculture and Agri-Food Canada, Lethbridge, AB; 2University of Guelph, \\ Guelph, ON; ${ }^{3}$ University of British Columbia, Vancouver, BC, Canada
}

Through advances in biotechnology, an array of technologies with the potential to improve production in the feedlot is developing. The enzymatic "machinery" required for digestion of the complex carbohydrates in grains and forages has been partially defined, and supplemental enzyme preparations designed to complement the microbial enzymes produced in the rumen are being engineered. Biotechnological advances in fermentation technology have lowered the cost of enzyme production to the point where the use of enzymes in commercial production is in the real of economic feasibility. Current evidence suggests that the microbial populations of the rumen may play an important role in the development of these commercial preparations, because they constitute a rich source of genes coding for highly active fibrolytic and amylolytic enzymes. Methods have been developed for several of the "key" ruminal bacteria for insertion of modifed or foreign genes into their genomes. Strategies to promote the survival of genetically modified bacteria in the rumen have been devised, although few have been tested. Alternatively, the problems associated with establishment of recombinant bacteria in the rumen may be circumvented by inducing the expression of desirable genes in the feed or by the animal itself. Although commercialization of these technologies will likely require eight to ten years, the foundation for their development has already been expressed in tobacco plants and the technology for expression of selected genes in ruminant feeds (e.g., barley, corn, soybean, canola) is rapidly being developed. Transgenic lambs have been developed which exhibit faster growth, greater disease resistance, and more desirable carcass traits than do their control counterparts. Given these remarkable advancements, there remains little doubt that biotechnology will play an increasingly important role in feedlot production. 